\title{
Gating neural development and aging via nuclear pores
}

\author{
Guang-Hui Liu ${ }^{1, *}$, Mo Lì ${ }^{2, *}$, Jing $\mathrm{Qu}^{1,2, *}$, Juan Carlos Izpisua Belmonte ${ }^{2,3}$ \\ ${ }^{I}$ National Laboratory of Biomacromolecules, Institute of Biophysics, Chinese Academy of Sciences, Beijing 100101, China; ${ }^{2}$ Gene \\ Expression Laboratory, Salk Institute for Biological Studies, 10010 North Torrey Pines Road, La Jolla, California 92037, USA; \\ ${ }^{3}$ Center for Regenerative Medicine in Barcelona, Dr. Aiguader 88, 08003 Barcelona, Spain \\ Cell Research (2012) 22:1212-1214. doi:10.1038/cr.2012.35; published online 13 March 2012
}

\begin{abstract}
Emerging evidence suggests an involvement of nuclear pore components in the regulation of neural differentiation and aging. These findings will have far-ranging impacts on the understanding of the function of the nuclear envelope in physiological settings and in various neurological diseases.
\end{abstract}

Embedded in the nuclear envelope, nuclear pore complexes (NPCs) are multiprotein channels, whose primary function is exchanging molecules between the cytoplasm and the nucleus. NPCs are composed of about 30 different proteins termed nucleoporins or Nups. They are located at sites where inner and outer nuclear membranes are connected. Besides regulating nuclear transport, nuclear pores are implicated in gene regulation and chromatin organization. Developmental processes frequently involve reorganization of the nuclear lamina and nuclear pores [1-3]. Notably, nuclear lamina components have recently been shown to regulate neural development and homeostasis. For example, loss of B-type lamins, which are ubiquitously expressed nuclear lamina components, causes spe-

\footnotetext{
*These three authors contributed equally to this work.

Correspondence: Guang-Hui Liua ${ }^{a}$ Juan Carlos Izpisua Belmonte ${ }^{\mathrm{b}}$

aE-mail: ghliu@ibp.ac.cn

bE-mail: belmonte@salk.edu, izpisua@cmrb.eu
}

cific defects in brain development [4]. Recently, D'Angelo et al. [1] reported that the change of NPC composition by a single nucleoporin is critical for neural differentiation. The expression of a transmembrane nucleoporin Nup210 is upregulated during the differentiation of embryonic stem cells (ESCs) into neural progenitor cells. Consistently, knockdown of Nup210 significantly blocks neural differentiation. A requirement for Nup210 in myogenesis was also observed [1]. Surprisingly, this role of Nup210 in differentiation lies in its regulation on differentiation-associated genes, but is not attributed to any change in nucleocytosolic transport or localization of inner nuclear transmembrane proteins, although disruption of the latter has been implicated in aberrant stem cell differentiation [2].

Nup210 is not the only nucleoporin that regulates neural differentiation. Deficiency of Nup133, a structural subunit of NPC, in mouse ESCs was also reported to hamper the generation of post mitotic neurons [5]. In addition to nuclear pore components, the nuclear transport machinery has also been implicated in neural differentiation. For example, the switch of nuclear import receptor importin- $\alpha$ subtypes is critical for neural differentiation of mouse ESCs [3]. Despite the identification of these exciting links between nuclear pore and neural development, the underlying mechanisms still require further elucidation.

The involvement of the nuclear lamina in physiological and premature aging has been well established. Mutations of lamin A are linked to premature aging syndromes $[6,7]$. Dysregulation of lamin B1 was also suggested to cause cell senescence [8]. However, despite the close ties to the nuclear lamina, the role of nuclear pores in the regulation of aging remains largely unknown. An early clue connecting aging and NPCs came from a report by D'Angelo et $a l$., in which they found that NPCs in postmitotic neurons do not turn over and are remarkably long lived [9]. More recently, the same group provided additional evidence of extremely long-lived nuclear pore proteins in rodent brains. They performed pulse chase labeling in rat and then analyzed global protein turnover spanning several years with a sensitive MudPIT (multidimensional protein identification technology). They found that the long-lived nuclear pore components such as Nup107 and Nup160 have extremely long lifespans in neuronal nuclei (more than 1 year). These proteins, serving as scaffold components of NPCs, integrate into the nuclear membrane of neuronal cells and live as long as the organism, without mitotic renewal [10].

Enjoying such longevity comes at a price, however. Without mitotic turnover, these NPC components may become oxidized or damaged as a result 
of being exposed to aging-related toxic metabolites accumulated during the lifespan. Consequently, increased nuclear membrane permeability resulting from progressive deterioration of NPCs has been observed in aged neurons, where cytosolic proteins are leaked into nuclear compartments [9]. It is possible that these leaked/mislocalized proteins could result in the impairment of various cellular functions, such as chromatin organization and gene expression.

So far, little is known about the aging-related factors that trigger NPC dysfunction in neurons. They may include oxidative stress, which is one of the most important factors inducing neurodegeneration, cell senescence and organism aging [9]. In young cells, a normal nuclear envelope composition appears crucial for the defense against oxidative stress. For instance, lamin B1 is able to recruit the antioxidative transcription factor OCT1 and safeguard somatic cells from oxidative stress [11]. Upon cell senescence, lamin B1 is downregulated with a concomitant increase in cellular sen- sitivity towards reactive oxygen species (ROS) [8]. In the context of neuronal aging, increased oxidative stress could not only attack long-lived nuclear pore components [9], but also disrupt classic nuclear import and export by directly interfering with the activity of nuclear transport machineries [12].

Enhanced cellular nitric oxide (NO), which causes nitrosative stress, is also strongly associated with aging-dependent neuronal degeneration. NO can directly modify proteins via $S$-nitrosylation of certain cysteine residues. Recently, NO was shown to $S$-nitrosylate and positively regulate $\mathrm{CDK} 5$, whose activation triggers nuclear lamina dispersion and neurodegeneration [13, 14]. It would be interesting to examine whether NO can directly modify and regulate certain NPC components.

Interestingly, a crosstalk between nuclear pores and nuclear lamina under stress conditions has emerged. In the premature aging context, accumulation of progerin, a truncated lamin A mutant, results in compromised nuclear import, which is associated with decreased func- tion of the nuclear sumoylation pathway [15]. Interestingly, progerin accumulation also causes increased oxidative stress [16]. It should be emphasized that increased oxidative or nitrosative stress can result in repression of the global sumoylation profile [17]. This complex regulatory network orchestrated by oxidative stress may play an important role in the functional degeneration of the nuclear envelope.

On the other hand, nuclear pores also appear to employ a hemostatic feedback mechanism to defend against oxidative stress-induced nuclear envelope lesions. For example, NO can directly modify Cys528 and Cys585 of the nuclear export receptor CRM1, resulting in inhibition of the assembly of the CRM1-Ran-cargo export complex. As a result, Nrf2, a CRM1 cargo and a critical antioxidative transcription factor, accumulates in the nucleus and signals to increase cellular antioxidant capability [18]. Together, these studies suggest that nuclear pores, as a major functional part of nuclear envelope, may be deeply involved in the pathogenesis
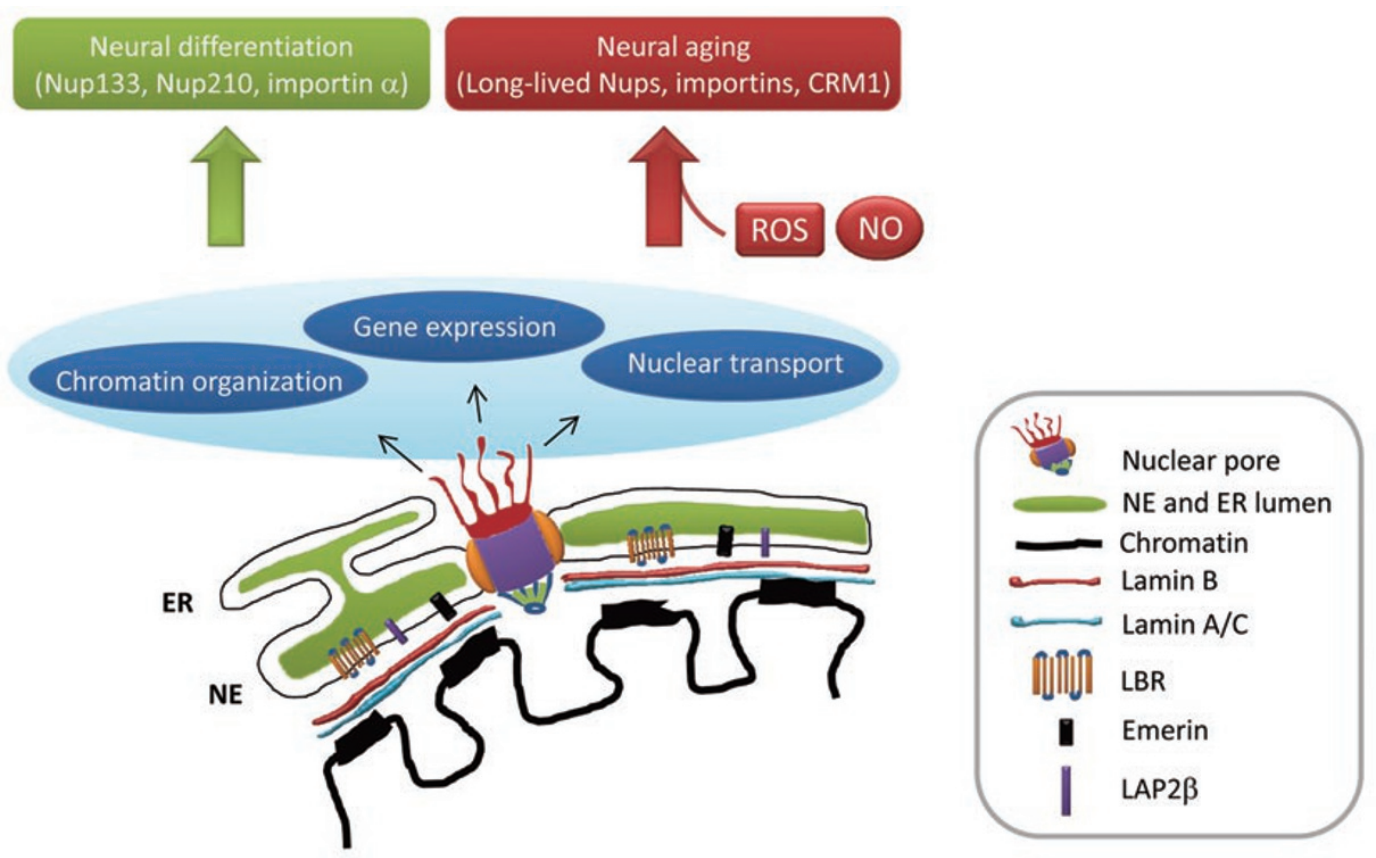

Figure 1 Summary of the emerging roles of nuclear pore in the regulation of neural development and aging. ER: endoplasmic reticulum; NE: nuclear envelope; ROS: reactive oxygen species; NO: nitric oxide. 
of aging-related neurodegeneration. Accrued damages to nuclear pores and nuclear lamina during the lifespan may culminate in a substantial abrogation of their function in safeguarding the intranuclear materials.

In summary, the emerging roles of nuclear pores in the regulation of neural differentiation and aging are exciting (Figure 1). Further revealing the mechanisms underlying these processes will indeed advance our understanding of various neurodevelopmental and neurodegenerative diseases.

\section{Acknowledgments}

GHL was supported by 100 Talents Program of Chinese Academy of Sciences and a Glenn aging foundation grant. JQ was partially supported by an AFAR/Ellison Medical Foundation postdoctoral fellowship. Work in the laboratory of JCIB was supported by Sanofi-Aventis, The Helmsley Charitable Trust and The Ellison Medical Foundation.

\section{References}

1 D'Angelo MA, Gomez-Cavazos JS, Mei A, Lackner DH, Hetzer MW. A change in nuclear pore complex composition regulates cell differentiation. Dev Cell 2012; 22:446-458.

2 Liu GH, Guan T, Datta K, Coppinger J, Yates J 3rd, Gerace L. Regulation of myoblast differentiation by the nuclear envelope protein NET39. Mol Cell Biol
2009; 29:5800-5812.

3 Yasuhara N, Shibazaki N, Tanaka S, et al. Triggering neural differentiation of ES cells by subtype switching of importin-alpha. Nat Cell Biol 2007; 9:72-79.

4 Kim Y, Sharov AA, McDole K, et al. Mouse B-type lamins are required for proper organogenesis but not by embryonic stem cells. Science 2011; 334:1706-1710.

5 Lupu F, Alves A, Anderson K, Doye V, Lacy E. Nuclear pore composition regulates neural stem/progenitor cell differentiation in the mouse embryo. Dev Cell 2008; 14:831-842.

6 Liu GH, Barkho BZ, Ruiz S, et al. Recapitulation of premature ageing with iPSCs from Hutchinson-Gilford progeria syndrome. Nature 2011; 472:221225.

7 Liu GH, Suzuki K, Qu J, et al. Targeted gene correction of laminopathyassociated LMNA mutations in patientspecific iPSCs. Cell Stem Cell 2011; 8:688-694.

8 Shimi T, Butin-Israeli V, Adam SA, et $a l$. The role of nuclear lamin B1 in cell proliferation and senescence. Genes Dev 2011; 25:2579-2593.

9 D’Angelo, MA, Raices M, Panowski SH, Hetzer MW. Age-dependent deterioration of nuclear pore complexes causes a loss of nuclear integrity in postmitotic cells. Cell 2009; 136:284295.

10 Savas JN, Toyama BH, Xu T, Yates JR, Hetzer MW. Extremely long-lived nuclear pore proteins in the rat brain.
Science 2012; 335:942.

11 Malhas AN, Lee CF, Vaux DJ. Lamin B1 controls oxidative stress responses via Oct-1. J Cell Biol 2009; 184:45-55.

12 Kodiha M, Stochaj U. Nuclear transport: a switch for the oxidative stresssignaling circuit? J Signal Transduct 2012; 2012:208650.

13 Qu J, Nakamura T, Cao G, Holland EA, McKercher SR, Lipton SA. S-Nitrosylation activates $\mathrm{Cdk} 5$ and contributes to synaptic spine loss induced by betaamyloid peptide. Proc Natl Acad Sci USA 2011; 108:14330-14335.

14 Chang KH, Multani PS, Sun KH, et al. Nuclear envelope dispersion triggered by deregulated $\mathrm{Cdk} 5$ precedes neuronal death. Mol Biol Cell 2011; 22:14521462.

15 Kelley JB, Datta S, Snow CJ, et al. The defective nuclear lamina in Hutchinsongilford progeria syndrome disrupts the nucleocytoplasmic Ran gradient and inhibits nuclear localization of Ubc9. Mol Cell Biol 2011; 31:3378-3395.

16 Richards SA, Muter J, Ritchie P, Lattanzi G, Hutchison CJ. The accumulation of un-repairable DNA damage in laminopathy progeria fibroblasts is caused by ROS generation and is prevented by treatment with $\mathrm{N}$-acetyl cysteine. Hum Mol Genet 2011; 20:3997-4004.

17 Qu J, Liu GH, Wu K, et al. Nitric oxide destabilizes Pias3 and regulates sumoylation. PLoS One 2007; 2:e1085.

18 Wang P, Liu GH, Wu K, et al. Repression of classical nuclear export by S-nitrosylation of CRM1. J Cell Sci 2009; 122:3772-3779. 